\title{
Discussion on Prefabricated Concrete Structure Design Method under BIM Technology
}

\author{
Wang Hongyan ${ }^{1}$, Zhang Zihong ${ }^{1}$ \\ ${ }^{1}$ Xi'an Traffic Engineering Institute, Xi'an Shaanxi 710077 China
}

\begin{abstract}
BIM is a data tool used in various stages of design, construction and management. It can integrate different models of buildings and realize sharing and transmission during the life cycle of the project, ensuring that engineering technicians can access different building information with a correct understanding and realtime response, it lays a good foundation for different participants including design, construction and management to achieve collaborative work. It is very important whether it is to ensure production efficiency, save costs and shorten construction periods. For the newly emerging prefabricated buildings, their structural design will become more efficient, accurate and reasonable due to the introduction of BIM technology.
\end{abstract}

\section{Overview of BIM technology}

BIM stands for building information model, which is based on 3D digital technology and integrates all the information of construction projects to form a complete data model, which can express project information in detail. The BIM model can store information of different professions, which is also an important foundation of this technology. BIM technology mainly uses the built BIM model to analyse the whole life cycle of the work of each stage of the project, and the entire analysis process can be visualized. As can be seen from the above introduction, BIM takes information as its core.

Autodesk Revit is a computer software developed based on BIM technology. It is the computer software with the best compatibility with the BIM platform at this stage. The advantage of this software is that it saves information in a three-dimensional model, and can produce different results according to needs during the design process, such as section, plan, and elevation. The information is consistent at different stages. If a certain place is modified, it can be reflected in every drawing and data related to it. In this case, the collaboration between technicians will become very convenient, which can effectively reduce or avoid repetitive work. The core meaning of BIM is not only three-dimensional model, but a brand-new management mode which takes BIM model as carrier (integrating relevant information of the whole construction industry cycle and the whole industry chain) and carries out engineering simulation construction by means of computer simulation. Through BIM Technology, the integration of various data and information in various stages of construction engineering survey, design, bidding and procurement, construction, operation and maintenance, update, demolition and other stages can be completed, and the simulation analysis of various data involved in the project construction process can be carried out, so as to complete the management and application of the whole life cycle of the construction project information.

At present, there is also a software dedicated to structural design, namely Autodesk Revit Structure. With the support of parameter-driven model, the structural design can be displayed in real-time in three dimensions, so as to accurately visualize different structures before structural construction.

\section{Features of BIM technology}

\subsection{Visualization}

From the perspective of the construction industry, the practical application of visualization plays an important role. For example, construction drawings mainly express the information of different components through the drawing of lines, and the specific structure form requires the staff to imagine. The introduction of BIM at this time can provide a brand new idea, namely visualization, so that components made up of lines become threedimensional entities ${ }^{[1]}$. Although in the current construction industry, there are some designs that can give renderings, but they only contain basic information related to components, and there is no interaction and feedback with other components. But the visualization of BIM can achieve interaction and feedback with other components. Because of the process of visualization, the results can be used to generate renderings, but also to enable all processes including design, construction, operation, etc. can be based on the visual environment. 


\subsection{Coordination}

In the construction industry, coordination is a key content. Not only the construction unit, but also the site team and the design team are carrying out coordination and cooperation. If a problem occurs during the implementation of the project, all relevant personnel need to organize a meeting to find out the cause of the problem, propose a solution, then change it and formulate remedial measures. In the design process, the lack of communication between different traits of designers often leads to professional collisions. The coordination performance of BIM solves this problem fundamentally. After the introduction of BIM, the possible collisions of all traits can be analysed and coordinated in the early stage of project and corresponding data can be generated. In addition to solve professional collision problems, it can also provide solutions for coordination between different sub-items and other designs.

\subsection{Simulation}

Simulation is not only the simulation design of the building model, but also the simulation of things that cannot be operated in the real world. In the design, BIM can be used to carry out simulation tests on all things that need to be simulated. In bidding and construction, the use of BIM can also achieve 4D simulation that is carry out construction simulation based on construction design, verify the feasibility of the plan through simulation, and provide correct guidance for construction. In addition, modern BIM can also achieve 5D simulation, that is, considering cost control on the basis of $4 \mathrm{D}$ simulation to achieve the expected cost control goal; in the later operation of the project, it can simulate the corresponding handling methods for different emergencies.

\subsection{Optimization}

The implementation process of the project is actually a process of continuous optimization. The introduction of BIM can achieve better optimization. Optimization will be directly affected by the three factors of time, information and complexity. If the information is inaccurate or insufficient, the optimization result cannot be given. For this reason, the BIM model can provide all the information for optimization; if the complexity is too high, it will too difficult for participants to grasp all the information using their existing capabilities, and other technologies and equipment are needed. The current modern buildings are basically far more complex than the maximum ability of the participants. Therefore, BIM supporting different optimization tools can achieve reasonable optimization of complex projects.

\subsection{Plotability}

For BIM models, other than conventional drawing there has various drawings, such as component processing drawings and architectural design drawings, you can also provide drawings of different professions through a series of processing of the target building, so that the expression of the project is more accurate and detailed.Through visual display, coordination, simulation and optimization of buildings, BIM can help owners to draw comprehensive pipeline drawings (after eliminating corresponding errors after collision inspection and design modification), comprehensive structure hole drawing (embedded casing drawing), collision inspection error detection report and suggestions for improvement.

Different from the conventional two-dimensional component processing drawing design, BIM Technology can use three-dimensional model to design prefabricated components, which can completely avoid the errors and omissions between components, and achieve the synchronous coordination between different disciplines and the factory processing personnel. After the component design is completed, the corresponding twodimensional drawing is directly derived according to BIM model. The two-dimensional drawing combined with BIM model can not only clearly convey the plane, vertical and sectional dimensions of traditional drawings, but also can clearly express the complex spatial combination relationship, so as to better ensure the perfect design and complete transmission of component processing information. Moreover, mobile devices such as mobile phones and tablet computers can be used for lightweight display of the model, and timely measurement can be carried out immediately.

\section{Housing industrialization and prefabricated buildings}

According to the conventional understanding, prefabricated building refers to the building constructed by reliable connection of prefabricated components. Prefabricated building has two main characteristics: the first feature is that the main components of the building, especially the structural components are prefabricated; the second is that the connection mode of prefabricated components must be reliable. According to the definition of the national standard "technical standard for prefabricated concrete building" (GB / T 51231-2016), prefabricated building is "a building in which the main parts of structural system, peripheral protection system, interior system, equipment and pipeline system are integrated with prefabricated components". This definition emphasizes that prefabricated buildings are the main part of four systems (not just structural systems), which are integrated with prefabricated components.

Housing industrialization is an important topic that has emerged in my country in recent years and has been recognized and vigorously promoted by the country. It refers to the use of factory production to construct housing and housing, and the housing and housing are regarded as a kind of factory product, with a unified structure. Based on technical standards, with the help of advanced production technology, the division of labour is carried out according to different professions, and then the process and produce of various components in the factory, and finally transported to the site to be assembled by 
mechanical equipment to form a complete residential building.

Prefabricated construction refers to the use of all prefabricated components produced in the factory. The transportation vehicles is used to transport prefabricated components to the site, and then these prefabricated components are assembled and connected on site ${ }^{[2]}$. Prefabricated buildings are the main development trend and direction of the construction industry for a long time now and in the future. It not only has the characteristics of industrialization and specialization, but also energy conservation and environmental protection, which is the only way for the construction industry to achieve sustainable development goals.

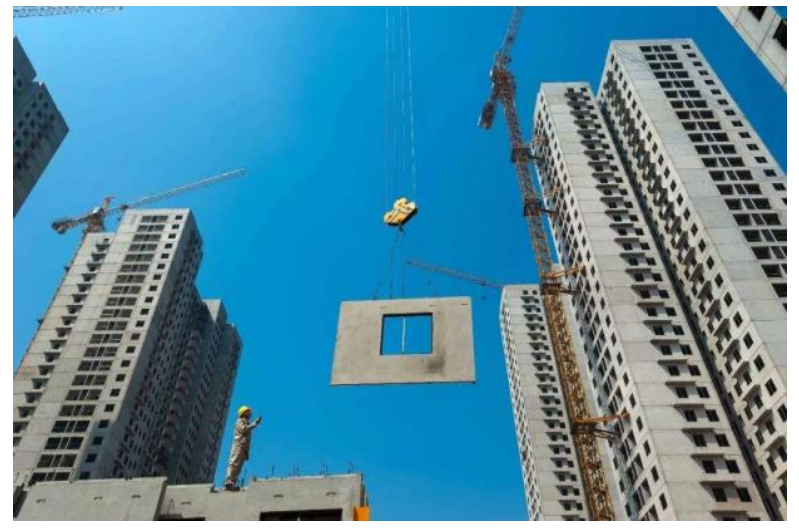

Fig.1 Construction drawing of prefabricated building

If you want to industrialize residential buildings, the key is to establish a complete house type library and component library, which can standardize the house type and component specifications of prefabricated buildings, avoid errors in design, and improve drawing efficiency to speed up actual work efficiency.

\section{BIM and prefabricated buildings}

The theories and ideas of BIM technology and prefabricated buildings have many common points and combinations. For prefabricated buildings, the main processes are: structural design, component production and on-site assembly. In the design, the content of plan design, preliminary design, construction drawing design and deepening design ideas are included. Among them, the use of deepening design in the production of prefabricated buildings is an important link for prefabricated buildings ${ }^{[3]}$. Builders analyse and study the composition of prefabricated building components, implement standardized design of components, and build a component library based on BIM technology while designing based on existing computer software. With the favourable support of BIM technology, the design of twodimensional components can be turned into threedimensional visualization.

In order to enable the rapid and stable development of prefabricated buildings, it is very important and necessary to introduce BIM technology to input the house type library and component library. In prefabricated buildings, the house types are formed by using prefabricated components. Based on this, the first thing to be solved is the component library, and then the house type library is established through different combinations to ensure design efficiency, reduce errors, and improve the drawing efficiency has laid a good foundation for the future development of prefabrication and mechanization.

\section{Prefabricated building structure design}

As mentioned earlier, Autodesk Revit Structure is a software dedicated to structural design. Therefore, take it as an example to analyse the design of prefabricated building structures. The production and production of prefabricated components is equivalent to taking part of the project construction to the factory environment for construction, so it is different from the traditional construction organization form. However, there are many similarities between the process and process of component processing and the traditional construction method, and there are many similarities in BIM management means.Component manufacturing and processing plants and construction units should prepare BIM components of embedded parts and lifting points required for component production and construction in advance, and carry out classification management in the form of component library. In the design stage, the corresponding BU components and design requirements are provided to the building quality and design department, and the design is integrated into the design, so that the architectural design is more accurate and feasible.

The application of BIM technology in prefabricated buildings has been generally recognized in the industry. The design of prefabricated building structures based on BIM can adopt the following methods: design according to the modularization and standardization of building components, and build after the integration of different technologies. The family library of components required for each structure, including prefabricated exterior wall panels, prefabricated beams and columns, prefabricated stairs and prefabricated floor slabs. When designing a specific structure, you can directly select standardized components from the above family libraries and compare different standardized components overlap each other to form a complete three-dimensional model, which can greatly speed up design efficiency ${ }^{[4]}$. In order to give full play to the design characteristics of prefabricated buildings, efforts should be made to research and develop different prefabricated building houses with modular characteristics to create good establishment of model libraries.

Through the continuous understanding and mastering of Autodesk Revit Structure, I understand that the family is very important for the actual use of Revit, and can define different types of settings and parameter variables based on the design of the family creator. In Revit, families can be divided into the following categories.

(1) Loadable families, also called component families, mainly include structural frames and basic components such as doors, windows, and columns. In the system, the component family file is an independent file, supports independent editing, and can be used multiple times in a 
variety of projects. After configuring extension plug-ins for the computer, using the above design method, the standardization and modular creation of fabricated building components can be carried out in the family template file, and then a family library of fabricated building structural components that can be used directly is formed. The file adopts ".rfa" "Format, in addition to being used as the detailed drawing of the structural components, drawing detailed and correct drawings for the actual component production, and downloading to the actual design process of the project under all necessary conditions, so as to realize the effective improvement of component design work efficiency.

(2) The system family is pre-defined in Revit and cannot be loaded from the outside. It can only be set or modified in the project. The walls, roofs, roofs and stairs of the building belong to the system family. All kinds of prefabricated components required for prefabricated buildings cannot be directly used to create a family library through the use of family template files. You can only open a project file in the format of ".rvt", and perform standardization and modelling requirements within the project. The prefabricated component is designed and then saved as a new project file in the format of ".rvt". The establishment of the component family library is completed. Using the above method of designing in the existing project files can form a detailed component library, and then provide detailed design for the production of various components in prefabricated buildings ${ }^{[5]}$. However, it should be noted that when designing a project, it cannot be loaded into the project at any time like a loadable family.

Since the component library corresponding to the system family can only be opened as a file in the format ".rvt" and cannot be loaded into the files corresponding to other projects, software developers need to seize this rare opportunity and increase research efforts. And through secondary development, the system family components including stairs, walls, boards, etc. are all established according to the requirements of modularization and standardization, which can be directly loaded in the project to speed up design work efficiency.

\section{Conclusion}

Most of the components of prefabricated building engineering are prefabricated and processed in the factory, and then connected and assembled on site. Not only is the industrial chain longer than that of ordinary buildings, but also due to the characteristics that prefabricated components cannot be dismantled and modified, the precision and complexity of each link of prefabricated construction engineering and management information are required to be high. Through the BIM Technology, the integrated management and information transmission of prefabricated building engineering data are combined with visibility, coordination and simulation. The five characteristics of optimization and drawing make the prefabricated building engineering realize collaborative management, visual design, digital production and fine construction, and then solve the management problems in the whole development and construction process of prefabricated building.

The fabrication of prefabricated components is the key link of prefabricated concrete building. According to the requirements of the construction period to produce highquality components and reduce the cost as much as possible, not only need the basic hardware configuration of the factory, but also need reliable technology and quantitative fine management. Factory managers should have a strong sense of planning, quantitative awareness, quality awareness and attention to details, with technical and management capabilities.

In summary, at present, my country's prefabricated construction is not mature enough, but many major groups have begun to study this aspect, and now they have achieved results in different links. Vigorously carry out the construction of prefabricated buildings, promote the application and development of BIM in them, and achieve the expected development goals as soon as possible.

Author: Wang Hongyan (1983-), woman, born in Qin'an, Gansu Province, Graduate degree, Senior engineer, teacher of Xi'an Traffic Engineering Institute. The research interests are measurement and valuation of construction projects.

\section{References :}

1. Wang Qihai. (2020) Discussion on the application of BIM technology in construction engineering cost management. Jushe, 15: 145

2. Yang Peng. (2017) Analysis on the design method of prefabricated concrete structure based on BIM technology. Housing and Real Estate, 3: 190.

3. Fan Lijun. (2020) Research on Construction Technology of Assembled Steel Camouflage Color Steel Roof by BIM Technology. Construction technology, 48:508-509.

4. YE Fei, LI Zhengkun, LIANG Qiaozhen. (2020) Optimization of Visual Project Schedule Management Based on BIM. Journal of Taiyuan University, 38:51-56.

5. He Yanqing.(2020) Application of BIM technology in civil engineering cost control. Si Chuan Cement, 08:222-223. 